\title{
Dynamic Nonreciprocity in Loss-Compensated Piezophononic Media
}

\author{
Aurélien Merkel, ${ }^{1}$ Morten Willatzen, ${ }^{2}$ and Johan Christensen ${ }^{1, *}$ \\ ${ }^{1}$ Universidad Carlos III de Madrid, Avenida de la Universidad 30, 28916 Leganés, Madrid, Spain \\ ${ }^{2}$ Beijing Institute of Nanoenergy and Nanosystems, Chinese Academy of Sciences, \\ Xueyuan Road No. 30, Haidian District, 100083 Beijing, China
}

(Received 20 August 2017; revised manuscript received 1 February 2018; published 28 March 2018; corrected 6 April 2018)

\begin{abstract}
Violating time-reversal symmetry enables one to engineer nonreciprocal structures for isolating and rectifying sound and mechanical vibrations. Rectifying sound is commonly achieved in nonlinear media, but the operation is inherently linked to weak and distorted signals. Here, we show how a pronounced electron-phonon coupling in linear piezophononic media under electrical bias can generate full mechanical rectification of broad spectral width, which permits the isolation of pulsed vibrations while keeping the wave-front shape fully intact. In this context, we deliberately show how the acoustoelectric effect can provide active loss compensation against lattice anharmonicity and thermoelastic damping. Further, our predictions confirm tunable nonreciprocity at an ultralarge contrast ratio, which should open the doors for future mechanical diodes and compact ultrasonic transducers for sensing and imaging.
\end{abstract}

DOI: 10.1103/PhysRevApplied.9.034033

\section{INTRODUCTION}

In conventional media, sound travels symmetrically in the sense that when excited at point $A$, the resulting acoustic energy flux at any point $B$ is the same as it would be at $A$ if sound would have been launched from $B$. This symmetry is attributed to reciprocity and is attainable in conventional media that obey time-reversal symmetry such as outward or inward propagating surface ripples on a pond [1]. Engineering wave propagation nonreciprocity, that is, to rectify waves along a one-way path only is not just fascinating from a physics standpoint, but inevitable in countless areas including noise control, energy harvesting, and transducer technology in general [2,3]. Particularly, ultrasonic sensing and imaging technologies in industry and medicine could benefit from perfect acoustic insulation that would protect the emitting sensor from echoes of its own beam. Usually these systems are designed for relatively high frequencies in the $\mathrm{MHz}$ range, which immediately implies the need of compact but also tunable devices.

Nonreciprocal acoustic wave propagation can be realized in linear media by using moving fluids that serve as the fixed, i.e., time-irreversible biased field in providing true insulation. To realize pronounced insulation, high flow rates are required, which inevitably leads to turbulence and shock-wave generation $[2,4,5]$. Another strategy relies on tailoring space-time modulation of the involved material to generate nonreciprocity [6-11]. Carefully designed nonlinear macroscopic structures have been realized to exhibit nonreciprocity without

*Corresponding author. johan.christensen@uc3m.es breaking time-reversal symmetry. Such acoustic rectifiers rely on coupling a nonlinear frequency-converting medium to a phononic crystal, which permits the passing of the nonlinear signal along one direction only [12-17]. Recent advancements along this line report on the use of two coupled nonlinear resonators [18] and propagation asymmetry induced by gravity [19]. Besides the inherent frequency conversion of these devices, the nonlinear mechanisms introduce severe signal distortions and reduced signal strength. Recently, a fishbone mechanical metamaterial has been engineered that combines large nonlinearities and geometrical asymmetries in producing static nonreciprocity [20].

In this Letter, we demonstrate a fully dynamical approach towards complete mechanical rectification in a piezophononic medium (piezoelectric semiconductor) [21]. By taking advantage of the acoustoelectric (AE) effect in the smallsignal limit, we demonstrate a nonreciprocal linear mechanical isolator of large bandwidth, high contrast ratio, and high power transmission efficiency in the passing direction. Besides the possibility to realize active tuning of the piezophononic properties, we also explore how quasiundistorted pulsed wave fronts can be rectified and loss compensated, which opens horizons in acoustic signal processing and imaging in the absence of parasitic and destructive backscattering.

Our strategy rests on applying a dc electric bias on a piezophononic semiconductor that can lead to strong directional-dependent mechanical attenuation or gain as induced by the electron-phonon interaction. This phenomenon, also know as the AE effect, has been extensively studied with a particular emphasis on amplifying ultrasonic waves [22-33], and more recently, exploring non-Hermitian and parity-time 


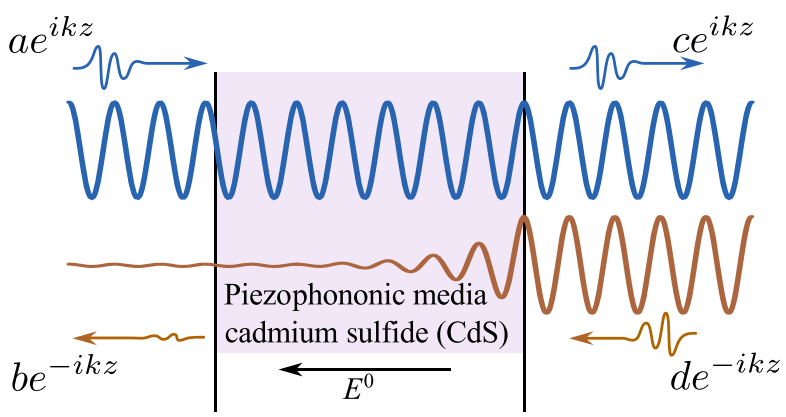

FIG. 1. A piezophononic layer acting as a highly nonreciprocal device when an electric field $E^{0}$ is applied. Maximal rectification $\sigma$ is reached when the ratio of opposite flowing energy fluxes is the largest. The approach is to let the forward flux (incoming from left, blue wave fronts) propagate undistorted and with high efficiency, while the backward flux (incoming from right, orange wave fronts) experiences very high attenuation. As a proof of principle, we take CdS to be the piezoelectric medium. The time convention is $e^{-i \omega t}$.

symmetric acoustics in solid media [34,35]. Our approach towards perfect nonreciprocity relies on the directional dependence of the electron-phonon interaction that is oddly symmetric on time reversal. In other words, elastic wave motion and electron drift (as dictated by the applied dc field) flow in parallel such that controlled amplification or attenuation can take place $[24,33,34]$. In this spirit, according to the Onsager-Casimir principle, $t^{R}\left(\omega, E^{0}\right) \neq t^{L}\left(\omega, E^{0}\right)$, which states that reciprocity is violated and directly linked to time-reversal asymmetry [36,37]. Here, $t^{R, L}, \omega$, and $E^{0}$ are the complex transmission coefficients across a finite region coming in from the left $(L)$ or right $(R)$, angular frequency, and applied electric field, respectively. It is desirable to acquire mechanical isolation at a high contrast ratio, defined as $\sigma \equiv\left|t^{L}\right|^{2} /\left|t^{R}\right|^{2}$, and high power transmission efficiency simultaneously. As illustrated in Fig. 1, our approach is based on tailoring mechanical wave propagation in a carefully biased piezophononic layer such that $t^{L} \rightarrow 1$ while $t^{R} \rightarrow 0$. In other words, we use the electron-phonon interaction to highly attenuate ultrasonic waves in one direction, while the amplitude opposing this direction remains quasiconstant. Our approach offers the major advantage that no frequency conversion takes place since no nonlinear acoustic effect is involved, therefore, there is no deformation of the acoustic spectrum. As we will show, a high rectification ratio can be obtained over an extended range of frequencies, in which several loss mechanisms can be compensated.

\section{MECHANICAL RECTIFICATION THROUGH THE ACOUSTOELECTRIC EFFECT}

We begin by considering the constitutive equations for piezoelectric materials

$$
\begin{aligned}
T_{i j} & =C_{i j k l} \epsilon_{k l}-e_{m i j} E_{m}, \\
D_{m} & =P_{\mathrm{sp}, m}+\varepsilon_{m j} E_{j}+e_{m j k} \epsilon_{j k},
\end{aligned}
$$

where $T_{i j}$ is the stress tensor, $C_{i j k l}$ is the elastic constant tensor, $\epsilon_{i j}=\left(\partial u_{i} / \partial x_{j}+\partial u_{j} / \partial x_{i}\right) / 2$ is the strain tensor, $e_{i j k}$ is the piezoelectric constant tensor, $P_{\mathrm{sp}, m}$ is the spontaneous polarization, $E_{i}$ is the electric field, $D_{i}$ is the electric displacement, and $\varepsilon_{i j}$ is the electric permittivity tensor at constant strain. The total electric field $E_{i}=E_{i}^{0}+E_{i}^{p}$ combines the externally applied field and a perturbation as of the linear elastic motion. The frequencydependent permittivity is deduced from the Drude model $\varepsilon_{i j}=\varepsilon_{i j}^{\infty} \varepsilon_{0}\left[1-\omega_{p}^{2} /\left(\omega^{2}+\tau^{-2}\right)\right]$, where $\varepsilon_{i j}^{\infty}$ is the highfrequency permittivity, $\varepsilon_{0}$ is the vacuum permittivity, $\omega_{p}=$ $\left[n_{0} q^{2} /\left(\varepsilon_{\infty} \varepsilon_{0} m_{\mathrm{eff}}\right)\right]^{1 / 2}$ is the plasma frequency, where $m_{\mathrm{eff}}$ is the electron effective mass, $\tau=m_{\mathrm{eff}} \mu_{\mathrm{dc}} / q$ is the carrier collision time, $q$ is the elementary positive charge, $n_{0}$ is the carrier density, and $\mu_{\mathrm{dc}}$ is the dc mobility. The constitutive relations in Eq. (1) are for isentropic conditions such that the Onsager relation applies. The electrical losses are accounted for by using a finite and frequency-dependent scalar complex carrier mobility $\mu_{n}=\mu_{\mathrm{dc}} /(1+i \omega \tau)$. The electron diffusion constant is found from the Einstein relation $d_{n}=\mu_{n} k_{B} T^{K} / q$, where $k_{B}$ is the Boltzmann constant and $T^{K}$ is the temperature in kelvin. The landscape of possible piezoelectric semiconductors is rather extended. In order to generate a strong phonon-electron coupling induced by the $\mathrm{AE}$ effect, we need a material that simultaneously has piezoelectric and semiconductor properties as provided with wurtzite structures, such as $\mathrm{ZnO}, \mathrm{GaN}$, and $\mathrm{CdS}$ [21]. Piezophononic media belonging to the wurtzite family provide strong piezoelectric coupling when an externally applied dc field and a longitudinal ultrasonic wave polarize along the hexagonal symmetry axis. As detailed elsewhere, combining the piezoelectric wave equation with the Maxwell-Poisson equation while conserving the electrical charge in the presence of an elastic perturbation, one arrives at the AE dispersion relation [24,35]

$$
\rho \omega^{2}=k^{2}\left\{C_{33}+\frac{e_{33}^{2}}{\varepsilon_{33}} \frac{1-\gamma \frac{k}{k_{0}}+i \frac{\omega}{\omega_{d}}\left(\frac{k}{k_{0}}\right)^{2}}{-\gamma \frac{k}{k_{0}}+i\left[\frac{\omega_{c}}{\omega}+\frac{\omega}{\omega_{d}}\left(\frac{k}{k_{0}}\right)^{2}\right]}\right\},
$$

where we have introduced the dielectric relaxation frequency $\omega_{c}=q n_{0} \mu_{n} / \varepsilon_{33}$, the sound velocity $v_{s}=$ $\left(C_{33} / \rho\right)^{1 / 2}$ with mass density $\rho$, the diffusion frequency $\omega_{d}=v_{s}^{2} / d_{n}$, the drift velocity of electrons $v_{d}=-\mu_{n} E_{z}^{0}$, the ratio of drift to sound velocities $\gamma=v_{d} / v_{s}$, and the dispersionless wave number $k_{0}=\omega / v_{s}$. Equation (2) can be recast into a fourth-order polynomial equation whose four roots represent four complex wave numbers for mechanical wave propagation-two in the forward direction, and two in the backward direction. Nevertheless, two of the roots have imaginary parts both with the same sign and order of magnitude as their real counterparts, which are independent of $E_{z}^{0}$ and frequency. These two 
roots correspond to highly attenuated waves and thus play only a minor role in both the electron-phonon interaction and the overall wave propagation. We are, therefore, left with only two wave modes, one corresponding to forward propagation $\left(k_{1}\right)$ and the other to backward propagation $\left(k_{2}\right)$, which can be expressed entirely analytically [29]. This result stems from the oddly symmetric electric bias $k_{1} \neq k_{2}$, which is an indication of broken time-reversal symmetry.

Equation (2) represents the $\mathrm{AE}$ dispersion relation for wurtzite structures for which we take cadmium sulfide $(\mathrm{CdS})$ as a common material choice with the following parameters: $T^{K}=293 \mathrm{~K}, \rho=4826 \mathrm{~kg} / \mathrm{m}^{3}, m_{\text {eff }}=0.27$, $C_{33}=93.6 \mathrm{GPa}, \quad e_{33}=0.44 \mathrm{C} / \mathrm{m}^{2}, \quad \varepsilon_{33}^{\infty}=9.4, \quad \mu_{\mathrm{dc}}=$ $0.0285 \mathrm{~m}^{2} \mathrm{~V}^{-1} \mathrm{~s}^{-1}$. The real parts of the wave numbers $k_{1}$ and $k_{2}$ remain similar to $\pm k_{0}$, while the imaginary parts differ substantially from each other when a bias electric field is applied. For the sake of presentation, however, we map these different modes on the same graph as shown in Fig. 2(a) by plotting $\operatorname{Im}\left[k_{1}\left(E_{0}\right)\right]=-\operatorname{Im}\left[k_{2}\left(-E_{0}\right)\right]$. Indeed, the drift current is unidirectional and the exchange of energy depends on whether the mechanical wave motion flows with the drift current or against it. When the drift velocity is equal to the sound velocity, which is the Cherenkov threshold $\gamma=1$, the imaginary part of the wave number whose direction coincides with the electric drift flow vanishes, which happens here for an applied electric field of $E_{z}^{0}=E^{0}= \pm 155.5 \mathrm{kV}$. This threshold marks the region where all forwardly directed mechanical vibrations propagate almost entirely unattenuated. This is our approach in the design of fully rectified mechanics comprising high power transmission efficiency at large isolation factors, which naturally requires a strong absorption in the opposite direction, preferably at a broad passing band. We emphasize the importance of the carrier density $n_{0}$ that can vary by several orders of magnitude, making the semiconductor behave like a resistive material or a conducting metal. In Fig. 2(b) we depict a constant and smooth absorption up to $n_{0} \leq 10^{13} \mathrm{~cm}^{-3}$ illustrating virtually dispersionless performance. However, as illustrated in Fig. 2(c), variations with frequency indicate that $\operatorname{Im}\left[k_{1}\right]$ exhibits gain despite electrically residing at the Cherenkov threshold. This deviation will later prove to be extremely advantageous when realistic losses are incorporated. The value of the carrier density can be modified, e.g., through the application of a variable electrostatic gating, by photoexcitation of the material, or simply by modifying the temperature, which in turn alters the electron-phonon interaction as seen in Fig. 2. To this effect, mechanical waves can be tuned towards perfect forward-directed intensity flux, while in reverse, mechanical attenuation can be enhanced with increasing doping levels of CdS. To assure a maximal contrast ratio $\sigma$ at that threshold, we seek for the spectral location $\omega_{\max }$ that provides a maximal attenuation. Over an extended range of frequencies, as
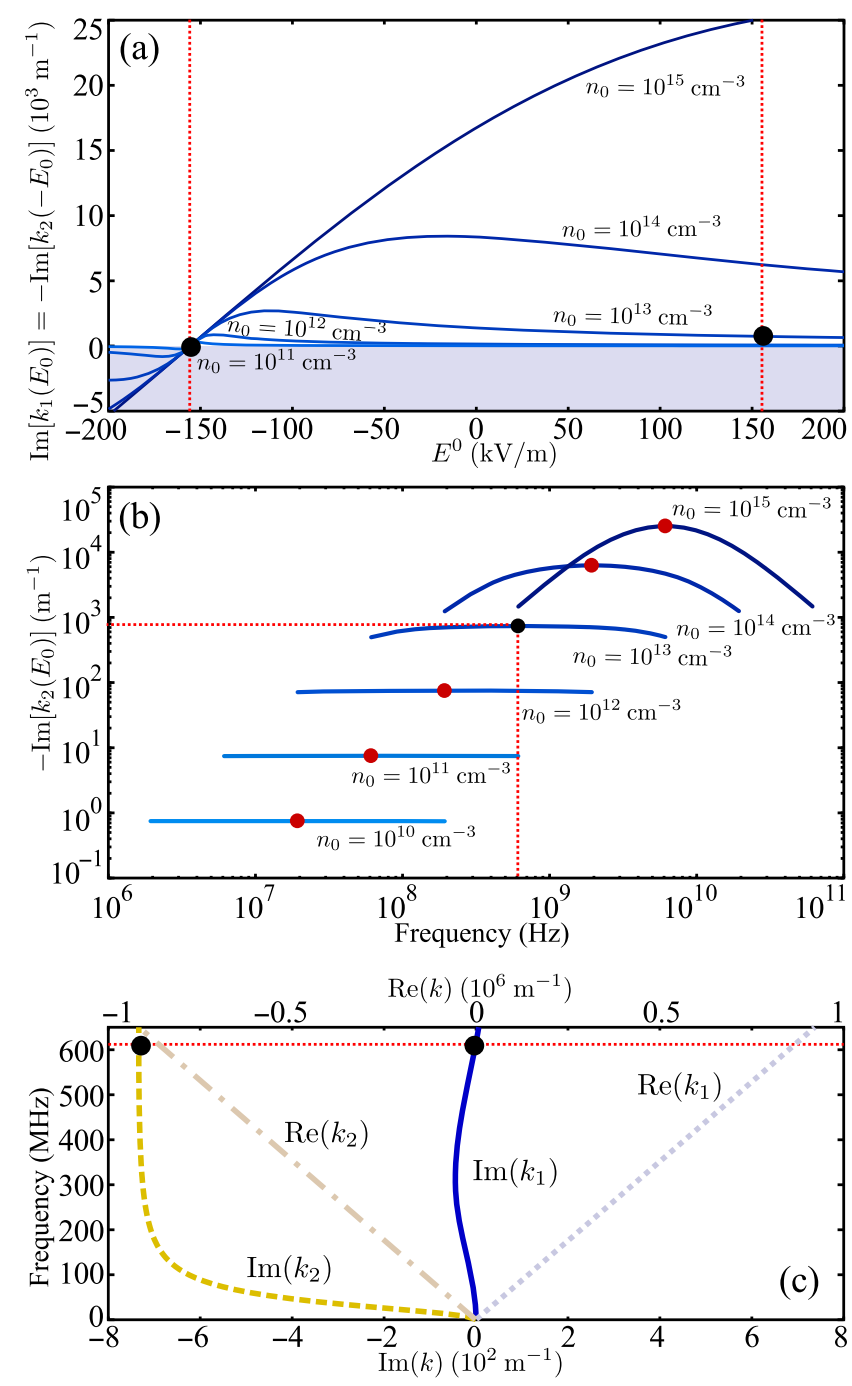

FIG. 2. Dispersion relation in dependence to the applied electrical dc bias $E^{0}$ in $\mathrm{CdS}$ as derived from Eq. (2). (a) Imaginary parts of the two wave numbers $k_{1}\left(E^{0}\right)$ and $k_{2}\left(-E^{0}\right)$ as a function of $E^{0}$ and for different carrier densities $n_{0}$ computed at their respective values of $f_{\max }\left(n_{0}\right)$. The gray area corresponds to the amplification zone, while the black dots mark the case for $n_{0}=10^{13} \mathrm{~cm}^{-3}$. (b) Spectral dependence of the attenuation $-\operatorname{Im}\left(k_{2}\right)$, where $f_{\max }\left(n_{0}\right)$ is indicated by red dots, at constant electrical bias $E_{0}=-155.5 \mathrm{kV} / \mathrm{m}$. (c) Complex wave numbers as a function of frequency at a fixed electric field $E_{0}=$ $-155.5 \mathrm{kV} / \mathrm{m}$ and $n_{0}=10^{13} \mathrm{~cm}^{-3}$. With correspondence to the previous panels, the red-dotted line marks the spectral region of maximal attenuation.

plotted in Fig. 2(c), we see that $\operatorname{Re}\left(k_{1,2}\right) \simeq \pm k_{0}$ since the imaginary parts of the wave numbers are much smaller than their real parts. Substituting this finding together with $\operatorname{Im}\left(k_{1}\right)=0$ into the dispersion relation in Eq. (2) gives us the possibility to find $\omega_{\max }$. More precisely, the strongest attenuation, that is, the maximum of $\operatorname{Im}\left(k_{2}\right)$, occurs when $\omega^{2}=\omega_{c} \omega_{d} \equiv \omega_{\max }^{2}$. In the low frequency regime, when $\omega \ll \tau^{-1}$, the electric permittivity can be reduced to 
$\varepsilon_{33} \simeq \varepsilon_{\infty} \varepsilon_{0}\left(1-\omega_{p}^{2} \tau^{2}\right)$ leading to the following approximated expression:

$$
\omega_{\max } \simeq v_{s} \omega_{p} \sqrt{\frac{m_{\mathrm{eff}}}{k_{B} T^{K}\left|1-\omega_{p}^{2} \tau^{2}\right|}} .
$$

Equation (3) remains valid in the range where $\omega_{\max } \ll \tau^{-1}$, which ensures that $\omega_{\max } \ll \omega_{p}$. In Fig. 2(c) we predict $f_{\max }=612 \mathrm{MHz}$, marking the region of maximal mechanical rectification, tuned at the exact vicinity of the Cherenkov threshold, $\gamma=1$. Further, in Fig. 2(b) we have indicated the spectral regions of maximum attenuation when increasing the carrier density $f_{\max }\left(n_{0}\right)$.

\section{SCATTERING PROPERTIES IN THE PRESENCE OF LOSSES}

We explore a pronounced mechanical nonreciprocity by tuning the forward flux toward lossless wave motion propagating in phase with the electrical drift, while ensuring sufficient attenuation in the opposite direction. With the same specifications given in Fig. 2, in the following, we demonstrate how a finite piezophononic CdS layer of $1-\mathrm{cm}$ width can act as an optimal mechanical isolator. We assume that the layer is impedance matched and electrically isolated to the surrounding. Upon fulfilling continuity of the displacements $u_{0, L}$ and the normal stresses $T_{0, L}$ at $z=0$ and $z=L$, we establish a connection among them across the layer through the impedance matrix

$$
\begin{aligned}
\left(\begin{array}{l}
u_{0} \\
u_{L}
\end{array}\right)= & \left(\begin{array}{ll}
Z_{11} & Z_{12} \\
Z_{21} & Z_{22}
\end{array}\right)\left(\begin{array}{l}
T_{0} \\
T_{L}
\end{array}\right)=\left(\begin{array}{c}
u_{0} \\
u_{L}
\end{array}\right) \frac{1}{e^{i k_{1} L}-e^{-i k_{2} L}} \\
& \times\left(\begin{array}{cc}
-\frac{e^{-i k_{2} L}}{i \Gamma_{1}}-\frac{e^{i k_{1} L}}{i \Gamma_{2}} & \frac{1}{i \Gamma_{1}}+\frac{1}{i \Gamma_{2}} \\
-\frac{e^{i\left(k_{1}-k_{2}\right) L}}{i \Gamma_{1}}-\frac{e^{i\left(k_{1}-k_{2}\right) L}}{i \Gamma_{2}} & \frac{e^{i k_{1} L}}{i \Gamma_{1}}+\frac{e^{-i k_{2} L}}{i \Gamma_{2}}
\end{array}\right)\left(\begin{array}{l}
T_{0} \\
T_{L}
\end{array}\right),
\end{aligned}
$$

where $\Gamma_{n} \equiv \rho \omega^{2} / k_{n}$, with $n=1$, 2. From the impedance matrix Eq. (4), we write the relation to the transfer matrix whose elements are related via

$$
\left(\begin{array}{ll}
M_{11} & M_{12} \\
M_{21} & M_{22}
\end{array}\right)=\left(\begin{array}{cc}
Z_{11} / Z_{21} & Z_{12}-Z_{11} Z_{22} / Z_{21} \\
1 / Z_{21} & -Z_{22} / Z_{21}
\end{array}\right)
$$

from which it is straightforward to derive the scattering matrix, whose elements, the scattering coefficients $t^{R, L}$ and $r^{R, L}$, relate the amplitudes of the incoming $(a, d)$ with the outgoing $(b, c)$ waves as illustrated in Fig. 1. In reality, however, vibrations in solids are always plagued by inherent and strong energy dissipation, which originates from lattice anharmonicity and thermoelastic damping. These frequency-dependent loss mechanisms are added to the imaginary parts of the wave numbers $k_{1,2}$ via the corresponding empirical attenuation coefficient, $\alpha_{\mathrm{CdS}}=$ $150 \mathrm{~dB} \mathrm{~cm}^{-1} \mathrm{GHz}^{-2}$ [38]. The transmittances for right and left incidence $\left|t^{R, L}\right|^{2}$, which are computed in Fig. 3(a),

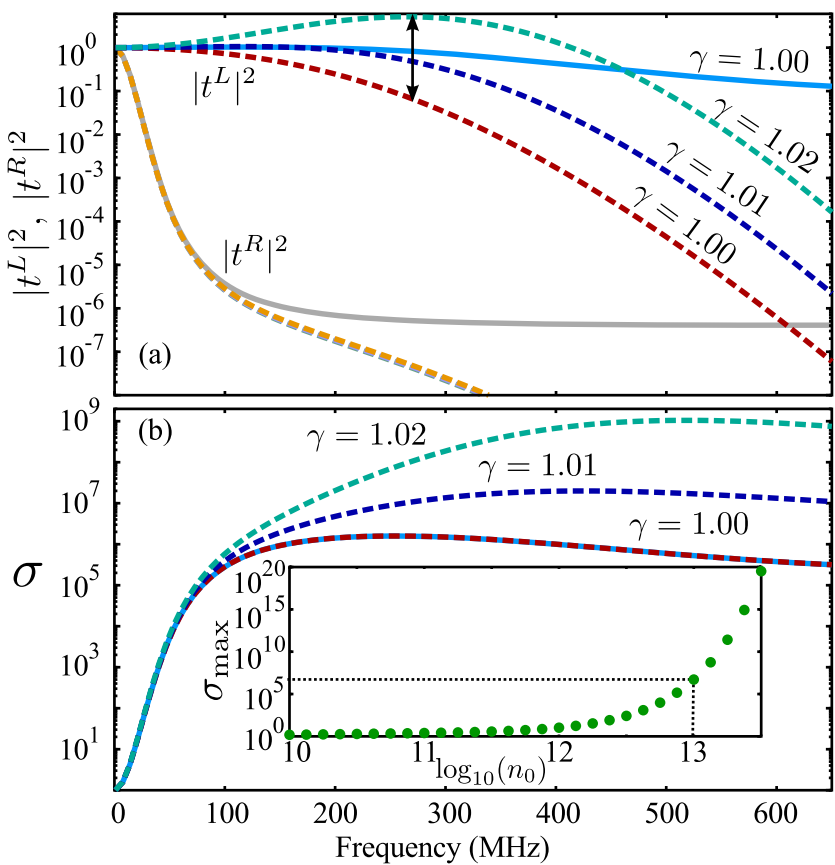

FIG. 3. Scattering properties of a piezophononic $\mathrm{CdS}$ layer (width $L=1 \mathrm{~cm}$ ), which is computed as a function of frequency, without losses, and under an applied electric field $E^{0}=-154.7 \mathrm{kV} / \mathrm{m}$ corresponding to $\gamma=1.00$ (continuous curves). When losses are present, we present three different scenarios: $E_{0}=-154.7,-156.2$, and $-158 \mathrm{kV} / \mathrm{m}$ corresponding to $\gamma=1.00,1.01$, and 1.02 , respectively (dashed curves). (a) Transmittance is plotted when ultrasound is incident either from right $(R)$ or left $(L)$ to the layer. (b) Spectral dependence of the contrast ratio $\sigma$. (inset) Maximum contrast ratio $\sigma_{\max }$ as a function of the intrinsic carrier density $n_{0}$ in units of $\mathrm{cm}^{-3}$, without losses.

differ substantially from each other and do, therefore, lead to an impressive contrast ratio above $\sigma=10^{5}$ extending over a large frequency band ranging from 100 to $400 \mathrm{MHz}$ as depicted in Fig. 3(b). As can be seen, not only is nonreciprocity intimately linked to time-reversal asymmetry, but an efficient transmittance in the forward direction $\left|t^{L}\right|^{2} \rightarrow 1$ guarantees high power transfer over a broad frequency range since we permanently reside at the Cherenkov threshold. When mechanical losses are introduced (dashed lines in Fig. 3), we predict a significant drop of the transmittance. To counteract on the losses, we increase the electric bias slightly, as specified in Fig. 3, such that the AE effect may compensate for them. In fact, for the case with $\gamma=1.02$, peaking at $260 \mathrm{MHz}$, we have reached an overcompensation of about $17 \mathrm{~dB}$, altogether showing that ultrasonic amplification in piezophononic media can minimize and eliminate losses and potentially be utilized in, e.g., lossy mechanical metamaterials similar to its optical counterpart [39]. Beyond that, as seen in the inset in Fig. 3, the maximum value of the contrast ratio can be further tuned by altering the carrier density of the piezophononic medium. 


\section{ONE-WAY GAUSSIAN PULSE PROPAGATION}

Given the broad spectral rectification window, we predict the isolation, and therefore the possible prevention of destructive backscattering from short ultrasonic signals, as shown in Fig. 4. The incident pulse $f(t)$, see Fig. 4(a), is a cosine function modulated by a Gaussian envelope, which is written as $f(t)=\cos \left(\omega_{0} t\right) e^{-t^{2} /\left(2 \sigma_{f}^{2}\right)}$, where $\omega_{0}$ is the central frequency of the Gaussian pulse and $1 / \sigma_{f}$ is the frequency half-width at $-3 \mathrm{~dB}$. The transmitted pulse $f_{R, L}^{t}$ is obtained through the inverse Fourier transform of $f(\omega)$, which is weighted by the corresponding complex transmission coefficients $t^{R, L}(\omega)$

$f_{R, L}^{t}(t)=\frac{\sqrt{2 \pi}}{2} \sigma_{f} F_{s} \int e^{-\left(\omega-\omega_{0}\right)^{2}\left(\sigma_{f}^{2} / 2\right)} t^{R, L}(\omega) e^{-i \omega t} d \omega$,

where $F_{s}$ is the chosen sampling frequency. In Fig. 4(b), it can be seen that the pulse in the passing direction (left incidence) is almost entirely undistorted after propagation through the layer with a time of flight $t_{f}=2.26 \mu \mathrm{s}$. Only marginally small differences, in view of the quasiconstant amplitude, can be discerned when compared to the incident wave front. Ultrasonic signals incident from the opposite direction are entirely blocked since the pulse amplitude approaches zero. This finding unequivocally shows that short microsecond pulses are efficiently rectified in piezophononic isolators as controlled through the external electric bias. We also stress that with slightly off-normal incidence angles strong rectification prevails over an extended frequency range [40].

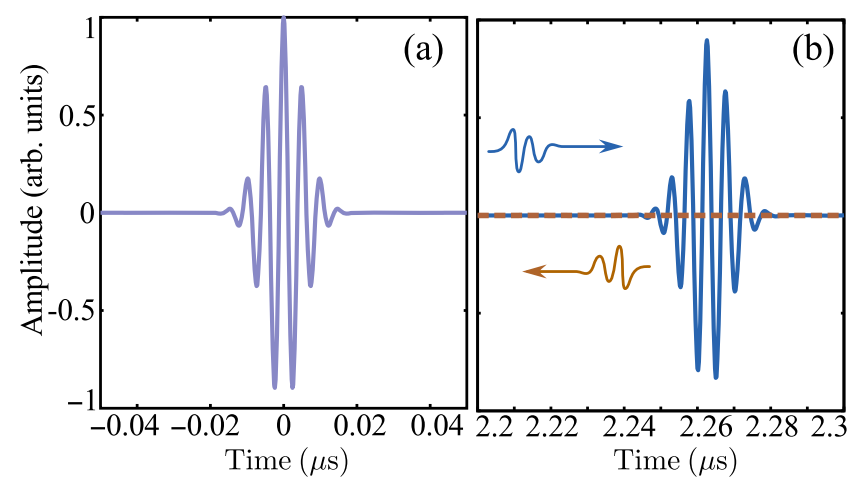

FIG. 4. Comparison of the temporal evolutions of a short ultrasonic microsecond pulse for right and left incidences. The piezophononic layer has the same specifications as in the previous example, with losses and $\gamma=1.01$, while the Gaussian pulse is centered at $200 \mathrm{MHz}$ and has a frequency bandwidth of $60 \mathrm{MHz}$. (a) Source signal. (b) Transmitted signals with left incidence (blue curve) and right incidence (orange curve). The amplitudes are normalized with the maximum amplitude of the source signal.

\section{CONCLUSION}

To conclude, we demonstrate dynamical nonreciprocity for linear mechanical waves. Based on the acoustoelectric effect in piezophononic media, we show that perfect isolation, that is, one-way transmission of ultrasonic pulses, stems from attenuationless and highly efficient forward propagation while waves are strongly absorbed in the opposing direction. This linear isolator is highly tunable and permits gigantic contrast ratios controlled through an externally applied electric field. We foresee that our findings can push forward engineering of ultrasonic transducers immune to backscattered pulse signals and provide a valid route towards loss-compensated mechanical metamaterials.

\section{ACKNOWLEDGMENTS}

J.C. acknowledges the support from the European Research Council (ERC) through the Starting Grant No. 714577 PHONOMETA and from the MINECO through a Ramon y Cajal grant (Grant No. RYC2015-17156).

[1] S. A. Cummer, J. Christensen, and A. Alù, Controlling sound with acoustic metamaterials, Nat. Rev. Mater. 1, 16001 (2016).

[2] R. Fleury, D. L. Sounas, C. F. Sieck, M. R. Haberman, and A. Alù, Sound isolation and giant linear nonreciprocity in a compact acoustic circulator, Science 343, 516 (2014).

[3] B.-I. Popa and S. A. Cummer, Non-reciprocal and highly nonlinear active acoustic metamaterials, Nat. Commun. 5, 3398 (2014).

[4] J. Christensen and M. Willatzen, Flow-induced resonance shift in sonic slit array metamaterials, Phys. Rev. B 85, 094304 (2012).

[5] Y. Aurégan and V. Pagneux, Slow sound in lined flow ducts, J. Acoust. Soc. Am. 138, 605 (2015).

[6] F. Li, C. Chong, J. Yang, P. G. Kevrekidis, and C. Daraio, Wave transmission in time- and space-variant helicoidal phononic crystals, Phys. Rev. E 90, 053201 (2014).

[7] N. Swinteck, S. Matsuo, K. Runge, J. O. Vasseur, P. Lucas, and P. A. Deymier, Bulk elastic waves with unidirectional backscattering-immune topological states in a time-dependent superlattice, J. Appl. Phys. 118, 063103 (2015).

[8] Qing Wang, Yang Yang, Xu Ni, Ye-Long Xu, Xiao-Chen Sun, Ze-Guo Chen, Liang Feng, Xiao-Ping Liu, Ming-Hui $\mathrm{Lu}$, and Yan-Feng Chen, Acoustic asymmetric transmission based on time-dependent dynamical scattering, Sci. Rep. 5, 10880 (2015).

[9] G. Trainiti and M. Ruzzene, Non-reciprocal elastic wave propagation in spatiotemporal periodic structures, New J. Phys. 18, 083047 (2016).

[10] C. Croënne, J. O. Vasseur, O. Bou Matar, M.-F. Ponge, P. A. Deymier, A.-C. Hladki-Hennion, and B. Dubus, Brillouin scattering-like effect and non-reciprocal propagation of 
elastic waves due to spatio-temporal modulation of electrical boundary conditions in piezoelectric media, Appl. Phys. Lett. 110, 061901 (2017).

[11] Daniel Torrent, William J. Parnell, and Andrew N. Norris, Loss compensation in time-dependent elastic metamaterials, Phys. Rev. B 97, 014105 (2018).

[12] N. Boechler, G. Theocharis, and C. Daraio, Bifurcationbased acoustic switching and rectification, Nat. Mater. 10, 665 (2011).

[13] Bin Liang, Bo Yuan, and Jian-Chun Cheng, Acoustic Diode: Rectification of Acoustic Energy Flux in One-Dimensional Systems, Phys. Rev. Lett. 103, 1043012009.

[14] Bin Liang, Xin-Ye Zou, Bo Yuan, and Jian-Chun Cheng, Frequency-dependence of the acoustic rectifying efficiency of an acoustic diode model, Appl. Phys. Lett. 96, 233511 (2010).

[15] B. Liang, X. S. Guo, J. Tu, D. Zhang, and J. C. Cheng, An acoustic rectifier, Nat. Mater. 9, 989 (2010).

[16] T. Devaux, V. Tournat, O. Richoux, and V. Pagneux, Asymmetric Acoustic Propagation of Wave Packets via the Self-Demodulation Effect, Phys. Rev. Lett. 115, 234301 (2015).

[17] A. Cebrecos, N. Jimenez, V. Romero-Garcia, R. Pico, V. J. Sanchez-Morcillo, and L. M. Garcia-Raffi, Asymmetric propagation using enhanced self-demodulation in a chirped phononic crystal, AIP Adv. 6, 121601 (2016).

[18] S. Lepri and G. Casati, Asymmetric Wave Propagation in Nonlinear Systems, Phys. Rev. Lett. 106, 164101 (2011).

[19] A. Merkel, V. Tournat, and V. Gusev, Directional asymmetry of the nonlinear wave phenomena in a three-dimensional granular phononic crystal under gravity, Phys. Rev. E 90, 023206 (2014).

[20] C. Coulais, D. Sounas, and A. Alu, Static non-reciprocity in mechanical metamaterials, Nature (London) 542, 461 (2017).

[21] Zhong Lin Wang, Piezotronics and Piezo-Phototronics (Springer-Verlag, Berlin, Heidelberg, 2012).

[22] A. R. Hutson, J. H. McFee, and D. L. White, Ultrasonic Amplification in CdS, Phys. Rev. Lett. 7, 237 (1961).

[23] A. R. Hutson and D. L. White, Elastic wave propagation in piezoelectric semiconductors, J. Appl. Phys. 33, 40 (1962).

[24] D. L. White, Amplification of ultrasonic waves in piezoelectric semiconductors, J. Appl. Phys. 33, 2547 (1962).

[25] F. S. Hickernell, The electroacoustic gain interaction in III-V compounds: Gallium arsenide, IEEE Trans. Sonics Ultrasonics 13, 73 (1966).

[26] F. S. Hickernell, The piezoelectric semiconductor and acoustoelectronic device development in the sixties, IEEE Trans. Ultrason. Ferroelectr. Freq. Control 52, 737 (2005).
[27] Vikrant J. Gokhale and Mina Rais-Zadeh, Phonon-electron interactions in piezoelectric semiconductors bulk acoustic waves resonators, Sci. Rep. 4, 5617 (2014).

[28] M. Willatzen and J. Christensen, Acoustic gain in piezoelectric semiconductors at $\varepsilon$-near-zero response, Phys. Rev. B 89, 041201(R) (2014).

[29] J. Christensen and M. Willatzen, Tunable broadband acoustic gain in piezoelectric semiconductors at $\varepsilon$-near-zero response, Acta Acust. Acust. 101, 986 (2015).

[30] M. Rotter, A. Wixforth, W. Ruile, D. Bernklau, and H. Riechert, Giant acoustoelectric effect in $\mathrm{GaAs} / \mathrm{LiNbO}_{3}$ hybrids, Appl. Phys. Lett. 73, 2128 (1998).

[31] Lei Shao and Kevin P. Pipe, Amplification and directional emission of surface acoustic waves by a twodimensional electron gas, Appl. Phys. Lett. 106, 023106 (2015).

[32] Z. Insepov, E. Emelin, O. Kononenko, D. V. Roshchupkin, K. B. Tnyshtykbayev, and K. A. Baigarin, Surface acoustic wave amplification by direct current-voltage supplied to graphene film, Appl. Phys. Lett. 106, 023505 (2015).

[33] H. Zhu and M. Rais-Zadeh, Non-reciprocal acoustic transmission in a GaN delay line using the acoustoelectric effect, IEEE Electron Device Lett. 38, 802 (2017).

[34] J. Christensen, M. Willatzen, V. R. Velasco, and M.-H. Lu, Parity-Time Synthetic Phononic Media, Phys. Rev. Lett. 116, 207601 (2016).

[35] J. Christensen, Coalescence towards exceptional contours in synthetic phononic media, Europhys. Lett. 114, 47007 (2016).

[36] A. A. Maznev, A. G. Every, and O. B. Wright, Reciprocity in reflection and transmission: What is a "phonon diode"?, Wave Motion 50, 776 (2013).

[37] R. Fleury, D. L. Sounas, M. R. Haberman, and A. Alù, Nonreciprocal acoustics, Acoust. Today 14, 11 (2015).

[38] M. F. Lewis, Attenuation of longitudinal ultrasonic waves in insulators at room temperature, J. Acoust. Soc. Am. 43, 852 (1968).

[39] S. Xiao, V. P. Drachev, A. V. Kildishev, X. Ni, U. K. Chettiar, H.-K. Yuan, and V. M. Shalaev, Loss-free and active optical negative-index metamaterials, Nature (London) 466, 735 (2010).

[40] See Supplemental Material at http://link.aps.org/ supplemental/10.1103/PhysRevApplied.9.034033 for details on modeling and computing the angular dependency to the piezoelectric parameters.

Correction: The email address was missing for the corresponding author at publication; the footnote has now been inserted. 